Copyright 2008 Society of Photo-Optical Instrumentation Engineers. One print or electronic copy may be made for personal use only. Systematic reproduction and distribution, duplication of any material in this paper for a fee or for commercial purposes, or modification of the content of the paper are prohibited.

DOI: http://dx.doi.org/10.1117/12.754802

\title{
Mechanism of coupling and interference in nano-slit
}

\author{
Bora Ung and Yunlong Sheng ${ }^{\mathrm{a}}$ \\ ${ }^{a}$ Centre d'optique, photonique et laser, Université Laval, Québec (QC), Canada G1K 7P4 \\ bora.ung.1@ulaval.ca,sheng@phy.ulaval.ca
}

\begin{abstract}
Electromagnetic diffraction of optical waves at a subwavelength aperture becomes a focus of recent research interest in order to explain theoretically and experimentally the transmission enhancement of arrays of nano-size holes in metallic screens. Some theories propose that the surface waves exited at a subwavelength aperture by diffraction is propagated to the neighboring aperture and interfered with the incident optical beam at that aperture, resulting in enhancing or suppressing of the transmitted energy through the aperture. In those theories one considers implicitly the interference of the two waves in the exit side of the aperture. However, in the entrance side of the aperture we note that the surface waves and the incident beam have perpendicular wavevectors and orthogonal polarizations before being coupled into a slit. It is then important to investigate how this interference occurs with mediation of mutual conversion between the surface waves and bulk waves at the nano-slit.

We find that a part of the surface wave energy scattered by the slit edge leaks into the slit and induces diagonal electrical charge dipole, which radiates new bulk waves and surface waves on the slit's side-walls. Multiple reflection Fabry-Perot resonators are formed in both horizontal and vertical directions over the slit, depending on the slit's width and depth. We demonstrate the hypothetic interference between the fields induced by the surface waves coupled into the slit and the normal incident wave, which induces also electric dipoles and new surface waves at the slit. Our calculation fits well with the experimental results. The work is significant for development of the optical diffraction theory on the metallic nano-size apertures.
\end{abstract}

Key words: Surface plasmons; Surface waves; Metals, optics of; Apertures; Interference.

\section{INTRODUCTION}

The extraordinary enhancement of the light transmission through subwavelength aperture arrays in metal films [1], or through a single hole surrounded by a number of grooves [2] excited enormous research interests. Numerous theories have been proposed to explain the transmission enhancement, resulting in new development of the fundamental theory of diffraction of the nano-sized metallic structures, where both the spatial features of the objects and the propagation distances are much smaller than the wavelength. In order to investigate the basic physical phenomenon one studies the simplest structure such as the young's two slits interference and the diffraction of a single slit [3-6]. Kowarz studied the contributions of both the homogeneous and evanescent waves to the field of diffraction [7]. Lezec et al. suggested an explicit model of subwavelength slit diffraction, considering the surface waves generated by the diffraction of the slit propagated on the surface from one slit to its neighboring slit. When arriving the next the surface wave would interfere with the normally incident beam at that slit, enhancing the transmission of the slit when the interference is constructive [8]. Both their theories are within the scalar diffraction theory framework. Schouten et al. proved this diffraction model by measuring the total intensity transmission of the two subwavelength slits as a function of the wavelength and the slit separation [3,9]. Their results can be explained by the interference of the surface plasmon polariton (SPP), which is excited at one slit and is propagated to another slit, with the incident beam at the slit. The interference is the source of modulation of the double-slit transmission. More experiments are report by Gay et al, which are explained by Lezec's theory [4]. Lalanne et al develop a theory of diffraction of a single nano-size metallic slit. This diffraction generates the SPP and a creeping wave in the nearby of the slit [5].

In all the works mentioned above, it is implicitly understood that the interference occurs at the exit of the nano-slit between the two waves that are generated by the normally incident wave and the incident surface wave, respectively. However, the mechanism of the coupling of the surface wave into the slit has not been yet addressed. We notice that before their arrival to the entrance of the slit, the impinging SPP and the normally incident beam have orthogonal polarizations and perpendicular wavevectors. It is then interested to investigate how the interference between them may

Holography and Diffractive Optics III, edited by Yunlong Sheng, Dahsiung Hsu, Chongxiu Yu Proc. of SPIE Vol. 6832, 68320E, (2007) - 0277-786X/07/\$18 - doi: 10.1117/12.754802 
occur. Generally speaking, one knows that there is a mediation mechanism at the nano-slit, which makes conversion between SPP and free-space bulk waves. However, the coupling mechanism of the SPP into the slit and the interference with the incident wave has yet to be fully understood.

In this paper we investigate the behavior of the SPP impinging on a metallic nanoslit, using the full vectorial solutions of the Maxwell equations with the finite-difference time-domain method (FDTD). In particular, we are interested in the mechanism of coupling a part of the impinging SPP into the slit and its interference with the normally incident beam. The FDTD has been used to model the optical transmission of subwavelength hole, array of slits and double-slits [10-12].

The scattering of SPP by surface defects or by discontinuities of the substrate's dielectric constant is a classical problem investigated by many authors [13-16]. It is well known that the incoming SPP to a surface defect or a material discontinuity is partially reflected, transmitted, and scattered into the free space above the metal surface. This scattering is relevant to probing of the SPP in near-field optical microscopy. However, to our best knowledge no detailed analysis has been reported on the coupling of the SPP into the slit, and the interference at the slit between the incoming SPP and the incident free-space beam.

In our analysis we find that the bulk waves radiated at the slit edge by scattering of the SPP leak into the slit and induce accumulated charges and new SPPs on the slit side-walls. A sole incident SPP can induce bulk wave multireflection resonance cavity modes between the two slit walls, and the SPP slit waveguide modes along the slit axis, with their field distributions depending on the slit's geometry. The addition of those modes with the slit waveguide mode induced by a single normally incident beam is equal to the field in the slit when the SPP and the plane wave beam are incident on the slit, simultaneously. Therefore, we confirm the hypothesis of the interference between the fields excited by the surface wave and by the normally incident wave at the entire space around the slit, but not only in the space after the slit exit. We demonstrate that the slit transmission is enhanced or suppressed, depending on the relative phases of the interfering fields.

\section{COUPLING SURFACE PLASOMNS POLARITONS INTO SLIT}

We consider an infinitely long slit perforated in a real metal film, such as silver, along the $z$-axis, beside which there is a groove of width $250 \mathrm{~nm}$ and depth $70 \mathrm{~nm}$. The surface plasmon polariton (SPP) is generated by a linearly p-polarized beam, with $E_{x}$ and $H_{z}$ components only in the free space, of wavelength $\lambda_{0}=500 \mathrm{~nm}$ normally incident on the groove. As shown on Fig. 1, SPPs are generated by diffraction in the groove and propagated away from the groove on the top metal surface in the $\pm x$ directions. The internal mechanism of the SPP generation in the groove is not our concern in this paper. Other SPP sources could also be used.

Consider the SPP propagating in the $+x$ direction on the film top surface and impinges on the slit from the left ridge. The distance $L$ from the groove top-right edge to the slit top-left edge is a parameter associated to the phase of the SPP incident on the slit. By choosing to vary $L$ instead to vary the wavelength, we avoid in this paper problems arising from the metal's dispersion.

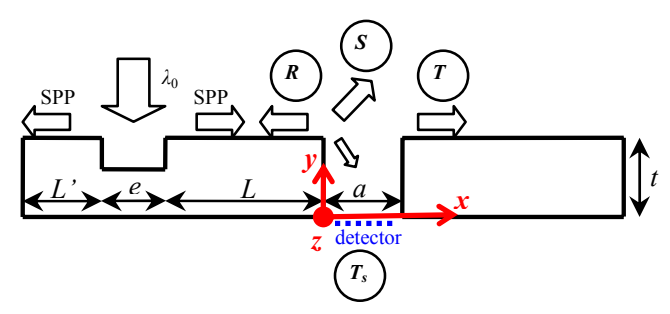

Fig. 1. Model schematic.

The FDTD is performed with a fine isotropic grid scale of $10 \mathrm{~nm}(\lambda / 50)$. The space discretization step $(\Delta x)$ is related to the time discretization step $(\Delta t)$ via the Courant equation: $\Delta t=S \Delta x$, where we choose is the Courant number $S=0.5$. The wavevector of the SPP is computed from the SPP dispersion relation: $k_{s p p}=n_{s p p} k_{0}$ where $n_{s p p}=\sqrt{\left(\varepsilon_{d} \varepsilon_{m}\right) /\left(\varepsilon_{d}+\varepsilon_{m}\right)}$ with $\varepsilon_{m}$ and $\varepsilon_{d}$ denoting the dielectric constants of the metal and adjacent dielectric medium, respectively. of the metal and the adjacent dielectric medium, respectively. The frequency-dependent complex permittivity of metal is described by the Lorentz-Drude model [19]: 


$$
\varepsilon(\omega)=\varepsilon_{r, \infty}+\sum_{k=0}^{K} \frac{f_{k} \omega_{p}^{2}}{\omega_{k}^{2}-\omega^{2}+j \omega \Gamma_{k}}
$$

where $\varepsilon_{r, \infty}$ is the dielectric constant at infinite frequencies, $\omega_{p}$ the plasma frequency, and $\omega_{k}, f_{k}$, and $\Gamma_{k}$ are the resonance frequency, strength and damping frequency, respectively, of $k$ th oscillator. The Lorentz-Drude model uses $K$ damped harmonic oscillators to describe the small resonances observed in the metal's frequency response. The values of the constants in Eq. (1) are taken from [11]. At $\lambda_{0}=500 \mathrm{~nm}$, we obtain the complex permittivity of silver: $\varepsilon^{=-}$ $7.6321+0.7306 i$. Perfectly-matched layer boundary conditions were applied at the limits of the computational domain [18]. The slit intensity transmission coefficient $T_{s}$ is calculated by integrating the time-averaged modulus of the Poynting vector along a plane "detector" located at the slit bottom exit $(0 \leq x \leq a, y=-10 \mathrm{~nm})$, as shown on Fig. 1.

We computed the electromagnetic fields and electrical charge distributions induced by the sole incident SPP for a set of slit width $a$ and thickness $t$ with the FDTD. The following is our observations on the results.

\subsection{Fabry-Perot resonator of SPP between groove and slit}

The incident SPP on the metal top surface on the air side is described by $H_{z}$ and $E_{y}$, which is parallel and normal to the top surface, respectively. At the slit top-left edge $(x=0, y=t)$, the abrupt change of permittivity of the material from metal to vacuum partially reflects the incident SPP. The remaining SPP is converted to bulk radiation by scattering. As the groove is present, the reflected SPP is again partially reflected back towards the slit from the top-right edge of the groove $(x=-L, y=t)$. The SPP characteristic propagation length [15] $L_{s p p}=1 /\left(2 \cdot \operatorname{Im}\left(k_{s p p}\right)\right)=5.2 \mu m$ is significantly longer than the propagation distance $L$ in our experiments. Thus, the multiple reflections on the section $-L<x<0$ of the metal top surface create a Fabry-Perot (F-P) type interference of the SPP. At the left-end ridge of the metal film the SPP propagating in the $-x$ direction is also partially reflected back. However, the propagation distance of the SPP in the $-x$ direction, $L^{\prime}$, is chosen to be $1.5 \lambda_{s p p}$ such that their interference is destructive and their perturbation to the $+x$ propagating SPP on the right-side of the groove is minimized.

\subsection{Bulk waves Fabry-Perot resonator modes between slit walls}

Of the bulk waves converted from the incident SPP at the slit top-left edge, a major part is scattered into the half-space above the metal film, see Fig. 1. However, our FDTD computation shows that a small part of the scattered divergent bulk waves leak into the slit. The bulk waves, mainly consisting of the $H_{z}$ and $E_{y}$ components, as for the incident SPP, hit and are reflected from the right-side-wall of the slit, and are then reflected back by the left-side-wall of the slit. The multiple reflections between the two slit walls form F-P resonator modes with slightly inclined bulk wave input from the top of the cavity. When the slit width is close to integer multiples of $\lambda_{0} / 2$, the cavity resonance creates strong standing wave fringes of $E_{y}$ and $H_{z}$ with a fringe period of $\lambda_{0} / 2$ as shown in Fig. 2(a) and 2(c). The fringes of $H_{z}$ are interlaced with the fringes of $E_{y}$. The maxima of $H_{z}$ are at the slit walls, while the maxima of $E_{y}$ are shifted by $\lambda_{0} / 4$ from the slit walls. These fringes represent the energy stored in the slit cavity. At the resonance, the slit's stored energy and transmission $T_{\mathrm{s}}$ are maximal. Out of the resonance, both $E_{y}$ and $H_{z}$ in the slit decrease to low intensities and $T_{\mathrm{s}}$ is minimal, as pictured in Fig. 2(b).

As shown in Fig. 2, the multiple reflection interference patterns of the scattered waves inside the slit overflow the top and bottom openings of the slit due to the weak confinement in this open cavity. The FDTD computation shows the new SPPs excited on both the top and the bottom surfaces of the metal film on the right-side of the slit $(x>a, y=0$ and $y=t)$. On the top surface, the SPP on the right-side of the slit is referred to as the transmitted SPP over the slit. This result is consistent with previous studies $[13,14,16]$. Indeed, when the slit is filled with another material of dielectric constant $\varepsilon_{2}$, or when the slit is replaced by a surface defect, such as a groove or bump of any shape, one can write down the analytic expressions for $H_{z}$ in the free space above the top surface $(y \geq t)$, which should satisfy the boundary conditions at the surface $y=t[13,14,16]$. When the slit of substrate $\varepsilon_{2}$ has a width $a>>\lambda_{s p p}$ the structure is referred to as a SPP Fabry-Perot interferometer. Its SPP energy transmission coefficient $T$ oscillates with period $\Delta a=\lambda_{s p p} / 2$ [16]. In the case of surface defects, Sanchez-Gil et al. found that the SPP intensity transmission coefficient $T$ across the defect exhibits a cavity-like oscillation with a period $\Delta a \sim 1.4\left(\lambda_{s p p} / 2\right)[13]$. They also predicted that incident SPP energy is partially stored in the groove [14]. Using the FDTD we are able to show the field in the slit below the interface as well as the energy stored inside the slit through F-P resonator modes.

Sanchez-Gil et al. showed in the case of a simple surface defect (groove or bump) that the scattering efficiency $S$ of the waves into the free space above the top surface depends on the SPP transmission $T$ (Fig. 1). When the surface defect is replaced by a slit, we observed that the scattering $S$ and its direction of maximum radiation both depend on the F-P 
resonator modes in the slit, which also determine the SPP transmission $T$ on the top surface and the slit transmission $T_{s}$ coefficients. The latter is the energy leaked from the slit's bottom exit in the form of the transmitted bulk waves and of the SPPs excited on the bottom plane of the metal film $(x<0$ and $x>a, y=0)$.

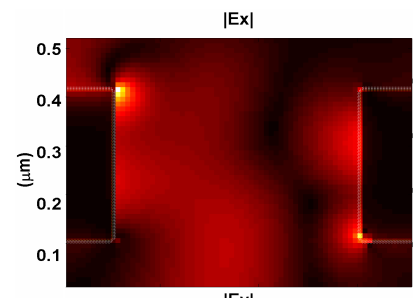

$|\mathrm{Ey}|$
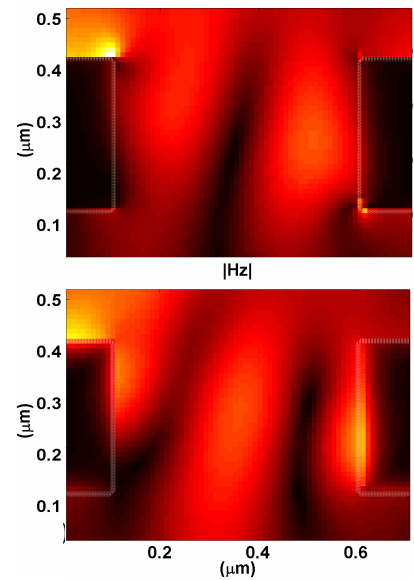

|Ex|

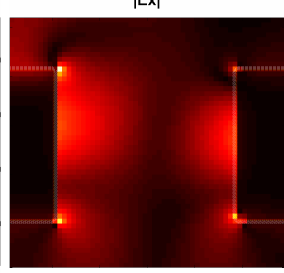

|Ey|
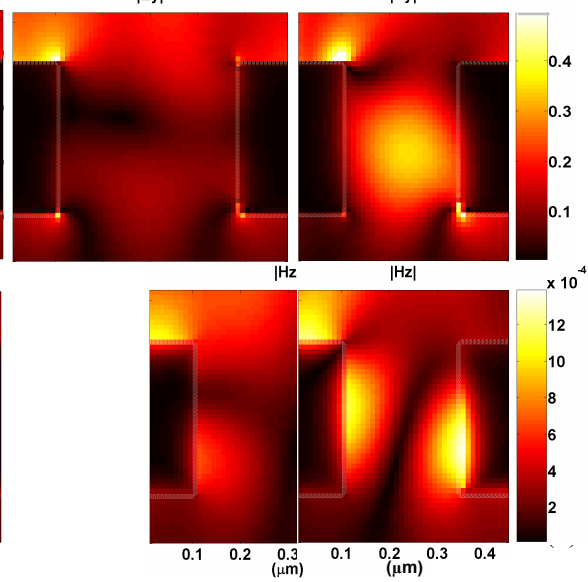

|Ex |

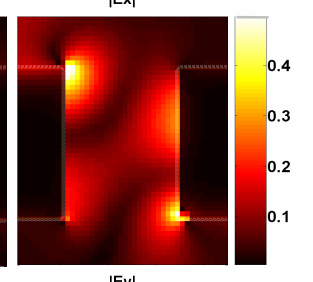

|Ey|

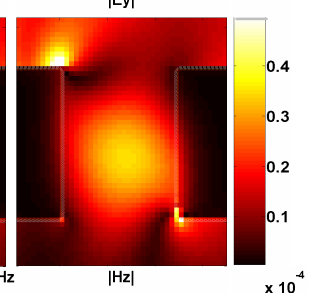

12

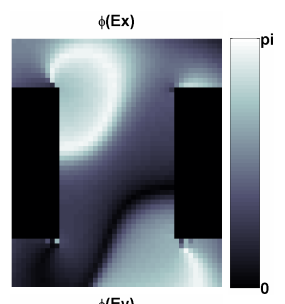

$\phi(E y)$
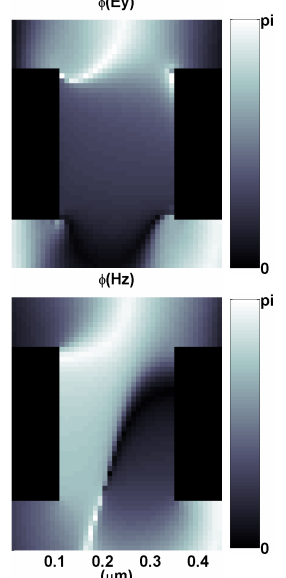

Fig. 2. Modulus of $E_{x}, E_{y}$, and $H_{z}$ when a single SPP is incident on a slit (a) width $a=500 \mathrm{~nm}$ at resonance; (b) $a$ $=380 \mathrm{~nm}$ out of resonance and (c) $a=250 \mathrm{~nm}$ at resonance. (d) Phase distribution for $a=250 \mathrm{~nm}$. In all figures, slit thickness $t=300 \mathrm{~nm}$ and SPP propagation distance $L=520 \mathrm{~nm}$.

\subsection{Excitation of new SPPs on slit walls}

As noted previously, the SPP on the air side of the top surface contains no $E_{x}$ component. The scattered bulk waves entering the slit contains $E_{x}$ which is responsible for the downward component of the bulk wave vector $\vec{k}_{0}$. In addition, new $E_{x}$ fields are excited on the slit walls. Indeed, the $H_{z}$ of the incident SPP induces surface currents parallel to the top metal surface $(-L \leq x \leq 0, y=t)$, while the bulk $H_{z}$ leaked into the slit induces surface currents parallel to the slit left-side wall $(x=0,0 \leq y \leq t)$. The surface currents associated with the incoming SPP must turn around the top-left corner $(x=0, y=t)$ as shown in Fig. 3. There is a corresponding sharp discontinuity in the amplitude of $H_{z}$ on the top surface SPP and that of the leaked bulk $H_{z}$, in the vicinity of the slit top-left edge, as shown in Fig. 2. According to the charge conservation equation, $\nabla \cdot \vec{J}=-\partial \rho / \partial t$, the corresponding current discontinuity creates the accumulation of electric charges at the slit corner as an oscillating monopole, which can be observed by a high concentration of $E_{y}$ on the top surface $(x \leq 0, y=t)$ and of $E_{x}$ on the slit left-side wall $(x=0,0 \leq y \leq t)$, as shown in Fig. 2. The oscillating monopole radiates waves which excites the SPP on the slit left-side wall $(x=0,0 \leq y \leq t)$.

On the right-side wall of the slit $(x=a, 0 \leq y \leq t)$, there is no significant $E_{x}$ when the slit width $a>>\lambda_{0}$. However, the scattered bulk $H_{z}$ induces surface currents and accumulated charges on the slit right-side wall within the skin depth. The FDTD calculation shows that the phase of $H_{z}$ is shifted by $\pi$ from the left-side wall to the right-side wall of the slit, as shown in Fig. 2(d), so that the surface currents are in the same direction along both walls. As a consequence, the accumulated charges on the slit right-side wall are of the opposite sign to that at the slit top-left edge, and the two poles are in-phase in their oscillation with time resulting in an oblique dipole across the slit as shown on Fig. 3. This dipole is the source of excitation of new SPPs on both slit walls, although the two poles can have different amount of charges, and the right-side pole is not necessarily located at the slit bottom-right corner but near the middle of the right-side wall. The distributions of field components $E_{x}$ and $H_{z}$ and of the accumulated charges on the two walls vary with the slit geometry and are asymmetric with respect to the slit axis, see Fig. 2(c). 


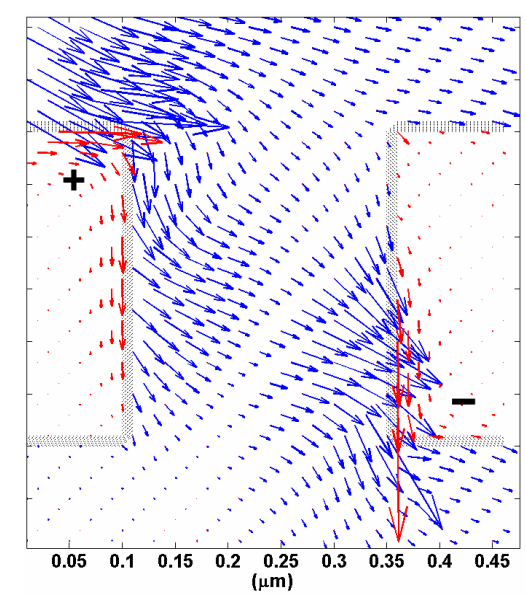

Fig. 3. Poynting $\vec{S}$ (blue arrows) and surface current density vectors $\bar{J}$ (red arrows) for single incident SPP illumination. The length of arrows is proportional to the amplitude. Signs $+/-$ indicate polarities of accumulated electric charges, which are related to the gradient of $\vec{J}$. Slit width $a=250 \mathrm{~nm}$, thickness $t=300 \mathrm{~nm}$.

The excited SPPs are sustained by the $E_{x}$ and $H_{z}$ on the slit side walls. In contrast with the $E_{y}$, whose maximum is shifted from the reflecting wall surfaces by $\lambda_{0} / 4$, the $E_{x}$ is stuck on the walls. Apparently, the multiple reflection resonances of the bulk waves between the two slit walls have little effect to the $E_{x}$ on the walls. When the bulk wave is out of resonance, $E_{y}$ is minimum inside the slit while the $E_{x}$ and $H_{z}$ components are non null on the slit walls, as shown in Fig. 2(b), where they sustain the SPPs on the slit side walls and contribute to coupling the incoming SPP on the top surface into the slit cavity.

\subsection{Coupling the incident SPP into the slit}

Figure 3 shows the Poynting vectors $\vec{S}$ associated with a single SPP incident on the slit. Since the magnetic field has only $H_{z}$ component, the direction of $\bar{S}$ is determined by the local ratio of $E_{x}$ and $E_{y}$. On the top surface, the $\bar{S}$ of the incident SPP is in $+x$ direction. The $\bar{S}$ then enters inside the slit by turning around the positively charged pole at the slit top-left edge due to the strong presence of accumulated charges at the edge. The $\bar{S}$ then flows downward supported by the excited SPP $\left(E_{x}\right)$ on the slit left-side wall. After propagating through a small depth in the slit, the magnitude of $E_{x}$ diminishes, and $\vec{S}$ changes the direction right conducted by the rising of $E_{y}$ in the middle of the slit, as shown in Fig. 2(c), to meet the right-side wall of the slit where the negatively charged pole and the excited SPP, sustained by $E_{x}$ and $H_{z}$, leads the $\vec{S}$ flux towards the bottom-right edge and to the slit bottom exit. In addition, new SPPs are excited on the bottom-right metal plane $(x \geq a, t=0)$.

\subsection{Fabry-Perot resonator modes along the slit axis}

It is well known that when a plane wave beam is normally incident on a nanoslit from free-space, the impedance mismatch between the region in the slit and the free-space region outside the slit is sufficiently important to allow strong F-P resonance modes along the slit axis [14]. Our FDTD computation shows that the incident SPP on the top surface may alone excite the slit waveguide modes for sufficiently thick films. When the slit width $a \leq \lambda_{0} / 4$, the $E_{x}$ distribution is oblique only at the top of the slit inside the first period, $\left(t-\lambda_{s p p}\right) / 2<y<t$, of the standing-wave fringes. As shown in Fig. 4(b), the standing-wave fringes of $E_{x}$ become uniform across the slit width for the remaining slit length, $0<y<\left(t-\lambda_{s p p} / 2\right)$, as in the conventional slit F-P waveguide modes for the slit illuminated by a normal incident beam.

\subsection{Field distributions inside the slit}

The pure bulk wave resonator modes between the two slit walls (horizontal F-P), as shown in Fig. 4(c), and the pure SPP resonator modes along the slit axis (vertical F-P), as shown in Fig. 4(b), represent the two extreme cases for wide and 
narrow slit, characterized by $a \geq \lambda_{0}$ and $a \leq \lambda_{0} / 4$ respectively. In general, a single incident SPP on the top surface induces 2D field distributions in the slit, which are asymmetric with respect to the slit axis.

Figure 4(a) shows the slit energy transmission $T_{s}$ normalized by the slit width $a$ as a function of slit width $a$ and thickness $t$, when a single SPP on the top surface impinges on the slit. Peak transmission occurs when both the vertical and horizontal F-P resonator modes are at resonance. Our computations show that the values of $a$ and $t$ for the resonances are shifted from those for the classical cavity resonances, $a=n\left(\lambda_{0} / 2\right)$ and $t=m\left(\lambda_{\text {eff }} / 2\right)$, where $m$ and $n$ are integers and $\lambda_{\text {eff }}=\lambda_{\text {spp }} / n_{\text {eff }}$ is the effective wavelength with slit waveguide mode effective index $n_{\text {eff }}$ [15]. For slit widths $a \leq \lambda_{0} / 4$, the F-P resonances along the slit thickness $t$ are clearly visible in Fig. 4(a), which occur when $t$ is $m\left(\lambda_{\text {eff }} / 2\right)$. The peak regions of $T_{s}$ have an inclined shape because $\lambda_{\text {eff }}$ and $n_{\text {eff }}$ are functions of the slit width $a$. With increasing of the slit thickness $t, T_{s}$ decreases due to attenuation losses of the SPPs' propagation along the slit walls. When the slit width $a$ is around $\lambda_{0} / 2$, the F-P resonances as a function of $t$ are still present, but with different shapes and locations of the $T_{s}$ peaks in the $a-t$ map due to the onset of bulk wave horizontal F-P modes. When the slit width $a$ is around $\lambda_{0}$ and $3 \lambda_{0} / 4$, the peaks of $T_{s}$ with $t$ dependence can still be seen in Fig. 4(a). However, the appearance of the resonances with variation of $t$ are not as regular as for small widths, $a \leq \lambda_{0} / 4$, because of the $2 \mathrm{D}$ asymmetric nature of the field distributions in the slit region and the weak confinement ability of the slit cavities for large widths $a$ and small thicknesses $t$.

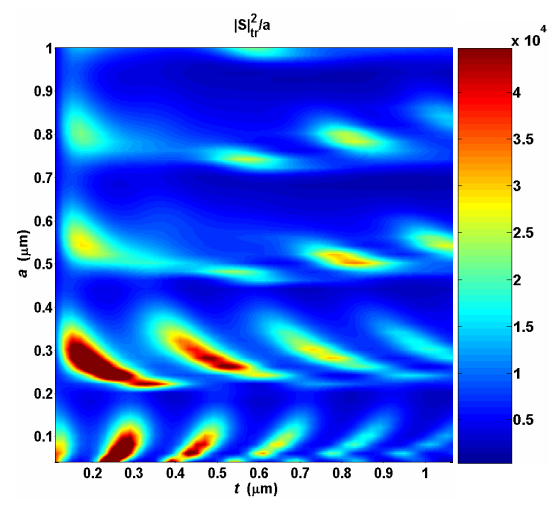

(a)

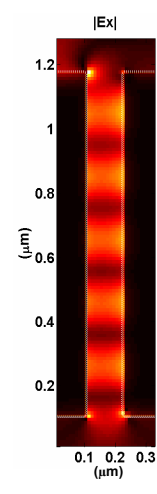

(b)

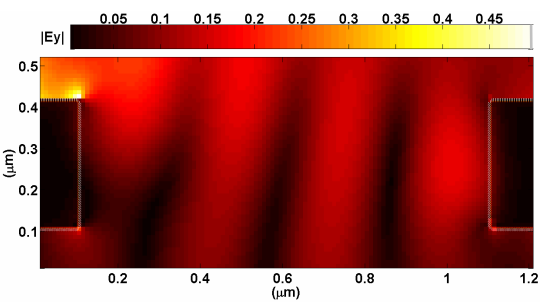

(c)

Fig. 4. Variation of (a) squared transmitted intensity normalized with slit width $a$ for various slit widths $a$ and thicknesses $t$ for a sole incident SPP. Standing wave fringes inside the slit in (b) vertical F-P modes $(a=120 \mathrm{~nm}, t=1 \mu \mathrm{m})$ and (c) horizontal F-P modes $(a=1 \mu m, t=310 \mathrm{~nm})$.

For a given slit thickness $t \sim \lambda_{s p p} / 2=235 \mathrm{~nm}$, the peaks of $T_{s}$ for the slit widths $a$ around $\lambda_{0} / 2, \lambda_{0}$ and $3 \lambda_{0} / 2$, are due to the horizontal bulk wave F-P resonances. The peaks at $a \sim \lambda_{0} / 2$ and $a \sim \lambda_{0}$ are spaced with an interval slightly larger than $\lambda_{0} / 2$.

\section{TRANSMISSION ENHANCEMENT AND SUPPRESSION BY INTERFERENCE}

We first computed the fields of $E_{x}, E_{y}, H_{z}$ and electrical charge distributions when a single $p$-polarized narrow beam is normally incident on the groove. As described in Section 2, this beam generates the SPP propagating on the top metal surface and impinging on the slit from the left side.

Then, we computed the field distributions when a single $p$-polarized narrow beam is normally incident on the slit. For this latter configuration, the FDTD results have been well described in $[5,6]$. The incident beam induces surface currents and accumulated electrical charges in the vicinity of the slit, which form two electric dipoles of nearly the same phase on the top and the bottom ridges of the aperture and parallel to the film surfaces. Fig. 5 shows the computed Poynting vectors, surface currents and induced electric dipoles in the vicinity of the slit for this case. The waves traveling along the slit axis form the F-P slit waveguide modes. In addition, the SPPs are excited and propagate away from the nanoslit in the $\pm x$ directions on both the top and bottom metal film surfaces.

We added the two individual field distributions by the instantaneous amplitudes of each component in the slit neighborhood, and then we computed the field distributions produced when the two beams are normally incident on the groove and the slit respectively and simultaneously. We found that the addition of the two individual field and charge distributions resulting from the two respective incident beams is identical to that resulting from the two simultaneously incident beams. Thus, we confirm that the interference between the normally incident beam and the incident SPP does 
occur around and inside the slit. This is natural since the structure responds linearly to the two individual incident beams when nonlinear effects are not taken into account.

The 2D field interference pattern on the top and bottom metal planes and inside the slit varies with the slit width $a$, thickness $t$ and the incident SPP propagation distance $L$, which controls the relative phase of the SPP-induced distribution with respect that induced by the normally incident beam. The slit transmission is enhanced by the incident SPP, when the two distributions are superposed constructively. It is reduced when the two distributions are superposed destructively.

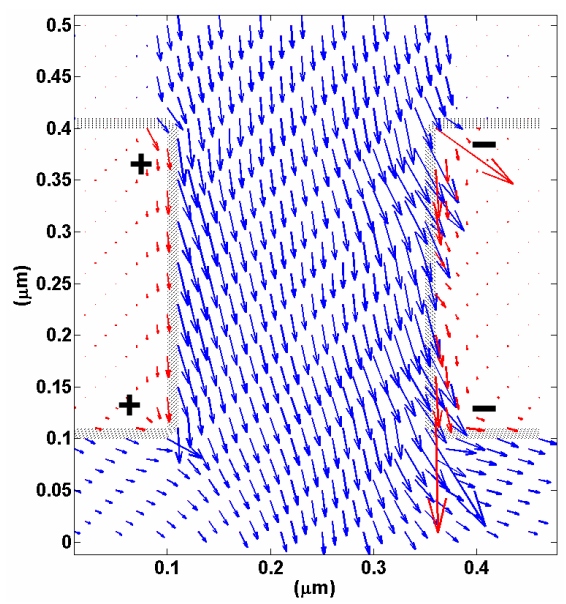

Fig. 5. Poynting $\bar{S}$ (blue arrows) and surface current density vectors $\vec{J}$ (red arrows) for a single normal incident beam on the slit $(a=250 \mathrm{~nm}, t=300 \mathrm{~nm})$. The length of arrows is proportional to the amplitude. Signs $+/-$ indicate polarities of accumulated electric charges.

Figure 6 shows the slit intensity transmission coefficient $T_{s}$ with the two incident beams, on the groove and on the slit, normalized by that of a single beam normally incident on the slit, as a function of the SPP propagation distance $L$. For constructive interference, the bare slit transmittance can be enhanced by more than 1.3 by the incident SPP on the slit. When the two distributions are superposed destructively, the transmittance can drop as low as 0.72 compared to the bare slit transmittance.

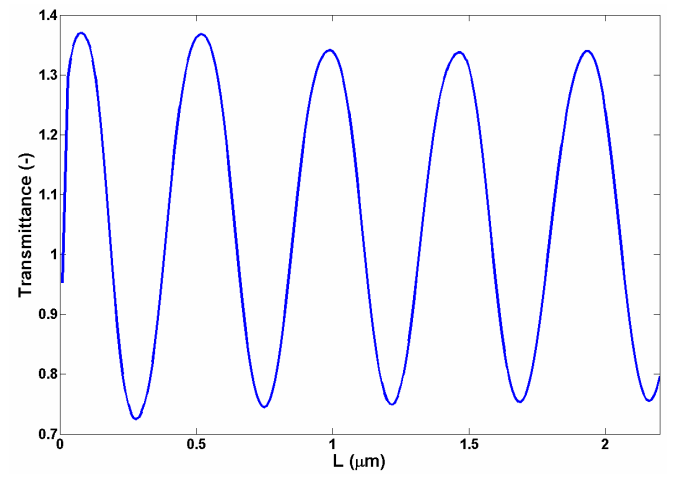

Fig. 6. Slit transmittance $T_{\mathrm{s}}$ (normalized on the transmission of a single normal beam on the slit) as a function of the slit-groove distance $L$. Slit parameters: $a=250 \mathrm{~nm}, t=300 \mathrm{~nm}$.

\section{CONCLUSION}

We have investigated the interference of the surface plasmon polariton (SPP) with an incident beam on a metallic nanoslit using the FDTD. The incident SPP on the film top surface is coupled into the slit by inducing oscillating electric charges at the slit edges which can emulate an oblique dipole that reradiates bulk waves inside and out of the slit, and 
excites new SPPs on the slit walls. The scattered bulk waves leaked into the slit form Fabry-Perot (F-P) resonator modes between the two slit walls. The excited new SPPs on the slit walls create the F-P resonator modes along the slit axis. The values of the slit width and thickness for the two types of cavity resonances are shifted from that of the standard F-P resonance conditions because of the asymmetric nature of the field distributions in the slit. We have demonstrated the interference between the impinging SPP and the normally incident beam by addition of their respectively induced modes in the slit through SPP/bulk-wave conversion. The slit transmission is enhanced or suppressed by the interference, depending on the relative phase between the incident SPP and the incident beam.

\section{REFERENCES}

1. T.W. Ebbesen, H.J. Lezec, H.F. Ghaemi, T. Thio, and P.A. Wolff, "Extraordinary optical transmission through sub-wavelength hole arrays," Nature (London) 391, 667-669 (1998).

2. T. Thio, K.M. Pellerin, R.A. Linke, T.W. Ebbesen, and H.J. Lezec, "Enhanced light transmission through a single subwavelength aperture," Opt. Lett. 26, 1972-1974 (2001).

3. h. f. Schouten et al. Plasmon-assisted two-slit transmission: Young's experiment revisited, Phys. Rev. Lett. 94, 053901 (2005)

4. G. Gay et al. The optical response of nanostructured surfaces and the composite diffracted evanescent wave model, Nature Physics, 2, 262267 (2006)

5. P. Lalanne and J. P. Hugonin, Interaction between optical nano-objects at metallo-dielectric interfaces Nature Physics, 2, 551-556 (2006)

6. B. Ung and Y. Sheng, Opt. Express. 15, 3, 1182-1190 (2007)

7. 7. M. W. Kowarz, "Homogeneous and evanescent contribution in scalar near-field diffraction", Appl. Opt. vol. 34, No. 17, 3055-3063 (1995)

8. H.J. Lezec and T. Thio, "Diffracted evanescent wave model for enhanced and suppressed optical transmission through subwavelength hole arrays," Opt. Express 12, 3629-3651 (2004).

9. H. F. Schouten, N. Kuzmin, G. Dubois, T. D. Visser, G. Gbur, P. F. A. Alkemade, H. Blok, G.W. Hooft, D. Lenstra, and E. R. Eliel, "Plasmon-Assisted Two-Slit Transmission: Young's Experiment Revisited," Phys. Rev. Lett. 94, 053901 (2005).

10. A.R. Zakharian, M. Mansuripur, and J.V. Moloney, "Transmission of light through small elliptical apertures," Opt. Express 12, 2631-2648 (2004).

11. Y. Xie, A.R. Zakharian, J.V. Moloney, and M. Mansuripur, "Transmission of light through slit apertures in metallic films," Opt. Express 12, 6106-6121 (2004).

12. Y. Xie, A.R. Zakharian, J.V. Moloney, and M. Mansuripur, "Transmission of light through a periodic array of slits in a thick metallic film," Opt. Express 13, 4485-4491 (2005).

13. J. A. Sanchez-Gil and A. A. Maradudin, "Near-field and far-field scattering of surface plasmon polaritons by one-dimensional surface defects," Phys. Rev. B 60, 8359-8367 (1999).

14. J. A. Sanchez-Gil and A. A. Maradudin, "Dynamic near-field calculations of surface-plasmon polariton pulses resonantly scattered at submicron metal defects," Opt. Express 12, 883-894 (2004).

15. H. Raether, Surface Polaritons on Smooth and Rough Surfaces and on Gratings (Springer-Verlag, Berlin, 1988).

16. T. A. Leskova, "Theory of a Fabry-Perot Type Interferometer for Surface Polaritons," Solid State Commun. 50, 869-873 (1984).

17. Y. Takakura, "Optical Resonance in a Narrow Slit in a Thick Metallic Screen," Phys. Rev. Lett. 86, 5601-5603 (2001).

18. A. Taflove and S. C. Hagness, Computational electrodynamics: the finite-difference time-domain method, 2nd edition, Artech House, Boston, 2000.

19. Aleksandar D. Rakic, Aleksandra B. Djuristic , Jovan M. Elazar, and Marian L. Majewski, "Optical properties of metallic films," Appl. Opt. 37, 5271-5283 (1998). 